\title{
Convenient multicomponent reaction synthesis of novel pyrano[4,3-b]pyran derivatives via a domino reaction under microwave irradiation
}

\author{
Jingpeng Mao, Jianqiang Wang*, Wenhui Zhang, Ziping Li, Jianlin Zhu, and Cheng Guo* \\ College of Chemistry and Molecular Engineering, Nanjing Tech University, \\ 30 Puzhu South Road, Jiangsu, Nanjing, 211816, China \\ E-mail:guocheng@njtech.edu.cn,jqwang@njtech.edu.cn
}

DOI: http://dx.doi.org/10.3998/ark.5550190.p009.398

\begin{abstract}
Novel pyrano[4,3-b]pyran-5-one derivatives have been obtained efficiently in a multicomponent domino cyclisation via the one-pot three-component reaction of aromatic aldehyde, 4-hydroxy-6methyl-2-pyrone and $\mathrm{N}$-methyl-1-(methylthio)-2-nitroethenamine in ethanol under microwave irradiation. The combined methods show excellent advantages such as higher yield, shorter reaction time and cleaner reaction, compared with conventional heating methods. The synthesized compounds are confirmed by ${ }^{1} \mathrm{H}$ NMR, ${ }^{13} \mathrm{C}$ NMR, IR and ESI-MS.
\end{abstract}

Keywords: Pyrano[4,3- $b]$ pyran-5-one, one-pot synthesis, multicomponent domino reaction, microwave irradiation

\section{Introduction}

The pyranopyran derivatives are an important class of heterocycle with a wide range of biological properties. ${ }^{1-4}$ Moreover, they possess important medicinal properties, such as antiproliferative, ${ }^{5}$ anti-inflammatory, ${ }^{6}$ antiviral $^{7}$ and anticancer. ${ }^{8}$ Some of the pyranopyran derivatives were reported to be used as the drugs, as shown in Figure 1. ${ }^{9-11}$

The development of new methodologies for the synthesis of pyranopyran is still a subject of considerable interest to synthetic and medicinal chemists. Consequently, a number of synthetic strategies for the construction of pyranopyran derivatives have been reported. ${ }^{12-16}$ However, the conventional multistep methods for the preparation of complex compounds result in synthetic inefficiency and the generation of large amounts of wastes because of complicated synthetic operations including extraction and purification in every step. Furthermore, many preparations of these compounds require long reaction times, ${ }^{17-18}$ harsh reaction conditions, ${ }^{19}$ and/or expensive catalysts or reagents. ${ }^{20}$ With the increasing requirement for new compounds for drug discovery 
in the fields of combinatorial medicinal chemistry, speed is very important to prepare novel compounds with the different structures. Thus, microwave-assisted organic synthesis approach is widely applied to achieve desired products because of shorter reaction times, high reaction selectivity and efficiency, compared with the classical approach. ${ }^{21-22}$ In addition, multicomponent reactions (MCRs) have attracted considerable attention from synthetic organic chemists for the construction of complex compounds because they represent an attractive synthetic strategy for rapid and efficient generation of compound libraries. ${ }^{23-24}$ MCRs can readily achieve high levels of diversity by simply changing the reacting components. ${ }^{25}$ Specially, onepot multi-component condensation via domino reaction supplies a possible route for an ideal synthesis, enabling the synthesis of complex molecules with maximum simplicity. ${ }^{26}$

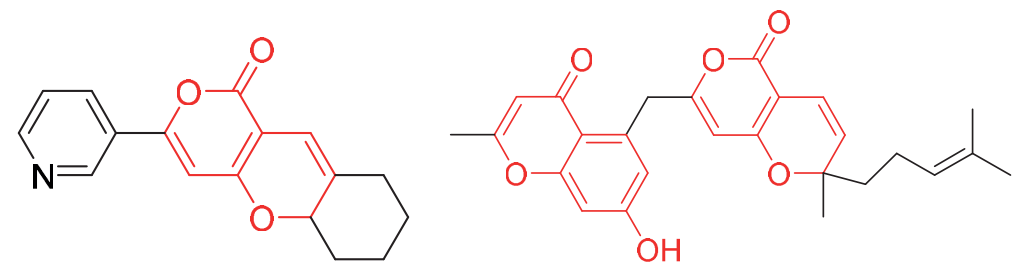

Antitumor Inhibitor of the HCT-116 human colon tumor cell line<smiles>CC(=O)CC(c1ccc([N+](=O)[O-])cc1)c1c(O)c2ccccc2oc1=O</smiles><smiles>CCOC(=O)COc1ccc2c(C)c(CCN(CC)CC)c(=O)oc2c1</smiles>

Acenocoumarol (antithrombus) Carbocromen (prevention of anginal attack)

Figure 1. Pyranopyran and chromene derivatives having biological activities. ${ }^{9-11}$

The reactant $N$-methyl-1-(methylthio)-2-nitroethenamine (NMSM) contains four active sites with three functional groups on an ethene motif, as shown in Figure 2. With a strongly electronwithdrawing nitro group, the nitroethylene substructure is a good Michael acceptor. The methylthio group is a good leaving group, and the methylamino group acts as electron donor, which makes the $\mathrm{C} 2$ a good Michael donor. Furthermore, So the compound of NMSM as an important reactant may cause the products more valuable as medical intermediate because the compound has two active groups of nitro and secondary amine compared with the other reactants. ${ }^{27-28}$ Overall, the ethylene moiety is a polarized push-pull alkene with $\mathrm{C} 1$ showing electrophilic character and $\mathrm{C} 2$ showing nucleophilic character. 


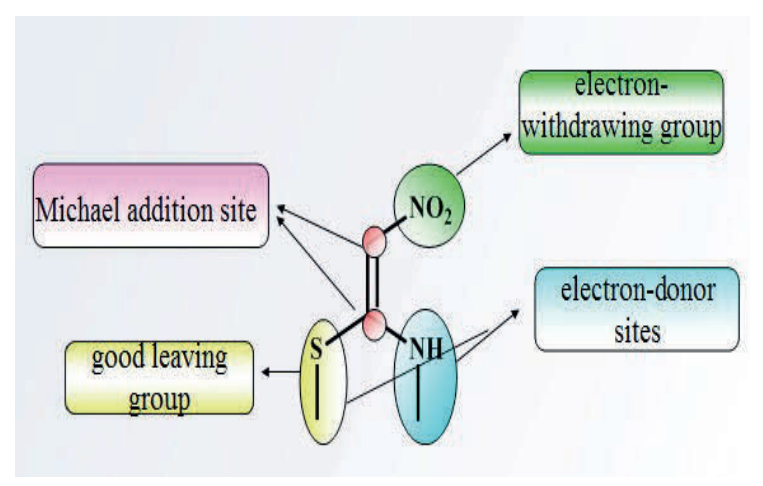

Figure 2. The reaction profile of NMSM.

Accordingly, we aimed to carry out an efficient and facile synthesis of pyranopyran derivatives by one-pot MCRs under microwave irradiation in a domino reaction. In this paper we report that the three components of aromatic aldehydes (1), NMSM (2) and 4-hydroxy-6-methyl2-pyrone (3) or 4-hydroxy-coumarin (5) can be used to produce novel pyranopyran derivatives $(4,6)$ in ethanol in the presence of ammonium acetate, as shown in Scheme 1. Besides, this synthesis followed the GAP chemistry (group-assisted-purification chemistry) ${ }^{29-31}$ process, which can avoid traditional chromatography and recrystallization purifications. Pure products can be obtained by washing the solid of crude products with cold ethanol. The structures of the synthesized compounds are confirmed by ${ }^{1} \mathrm{H}$ NMR, ${ }^{13} \mathrm{C}$ NMR, IR and ESI-MS.

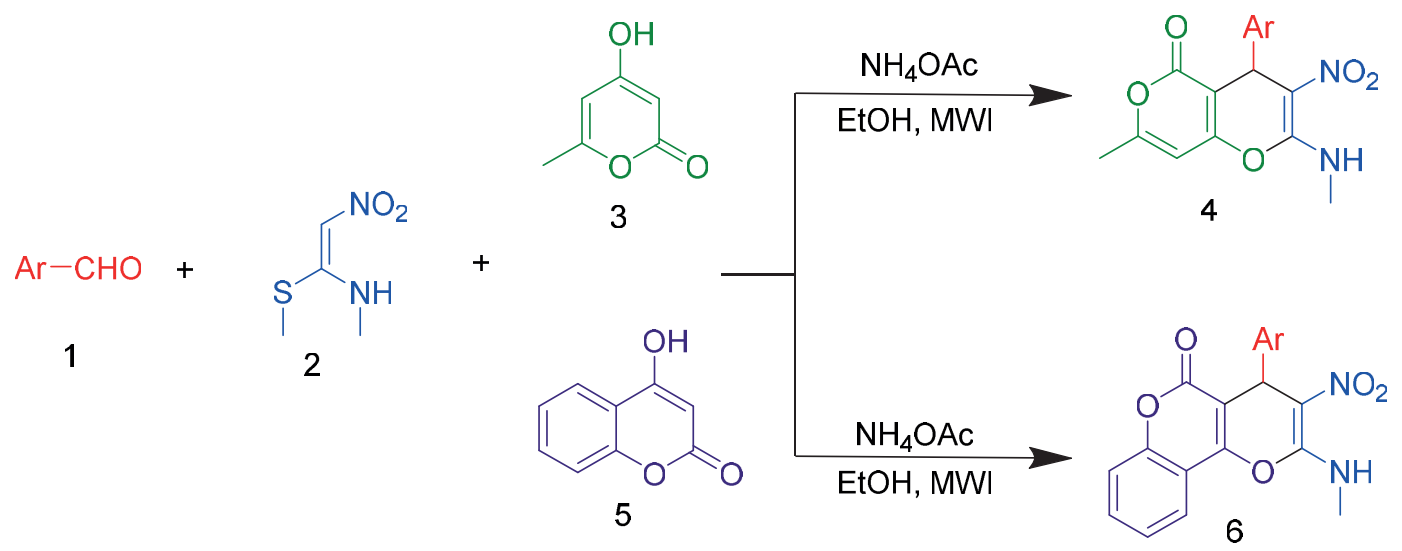

Scheme 1. Preparation of pyranopyran derivatives.

\section{Results and Discussion}

In initial experiments to optimize the reaction conditions, we used 4-methoxybenzaldehyde (1i), NMSM (2), and 4-hydroxy-6-methyl-2-pyrone (3) in ethanol at $80{ }^{\circ} \mathrm{C}$ under microwave 
irradiation, using different bases. The bases had a significant influence on the reaction results. When the reaction was conducted using different catalysts the reaction yields were widely variable. As shown in Table 1, the reaction in the absence of catalyst furnished a very low yield of $4 \mathbf{i}$ (Table 1, entry 1). And the reaction did not proceed in the presence of strong base (Table 1, entry 2). Besides we noticed that the other bases failed to catalyze the reaction efficiently (Table 1 , entries 3-7). It was however gratifying to discover that ammonium acetate catalyzes the reaction in ethanol efficiently, affording a maximum yield (70\%) of $4 \mathbf{i}$ (Table 1, entry 8).

In the reaction, the microwave energy is another factor which affects the yield of $4 \mathbf{i}$. Figure 3 indicates the relation between the yield of product $4 \mathbf{i}$ and the microwave irradiation power. The reaction was carried out under five different power settings: $100 \mathrm{~W}, 200 \mathrm{~W}, 300 \mathrm{~W}, 400 \mathrm{~W}, 500 \mathrm{~W}$. We find that the yield of $4 \mathbf{i}$ reached $61 \%$ when the microwave irradiation power was $100 \mathrm{~W}$, increasing to $70 \%$ when the microwave irradiation power was increased to $200 \mathrm{~W}$. However, if the power was higher than $200 \mathrm{~W}$ the yield of product $4 \mathbf{i}$ fell. This result suggested that higher microwave energy could result in a number of by-products. Moreover, the target product was probably decomposed by the higher energy. Therefore, according to the above analysis, the most suitable power for this reaction is $200 \mathrm{~W}$.

Table 1. The effect of different catalysts on the yield of pyranopyran $4 \mathbf{i}$<smiles>COc1ccc(C=O)cc1</smiles>

$1 i$<smiles>CN/C(=C\N=O)SC</smiles>

2<smiles>Cc1cc(O)cc(=O)o1</smiles>

3<smiles>CCOC(=O)CNC(N)=O</smiles>

\begin{tabular}{cccc}
\hline Entry & Solvent & Catalyst & Yield (\%) \\
\hline 1 & EtOH & - & 10 \\
2 & EtOH & $\mathrm{NaOEt}$ & Trace \\
3 & $\mathrm{EtOH}$ & $\mathrm{K}_{2} \mathrm{CO}_{3}$ & 10 \\
4 & EtOH & piperidine & 16 \\
5 & $\mathrm{EtOH}$ & $\mathrm{Et}_{3} \mathrm{~N}$ & 14 \\
6 & $\mathrm{EtOH}$ & L-proline & 15 \\
7 & $\mathrm{EtOH}$ & meglumine & 11 \\
8 & $\mathrm{EtOH}$ & $\mathrm{NH}_{4} \mathrm{OAc}$ & 70 \\
\hline
\end{tabular}




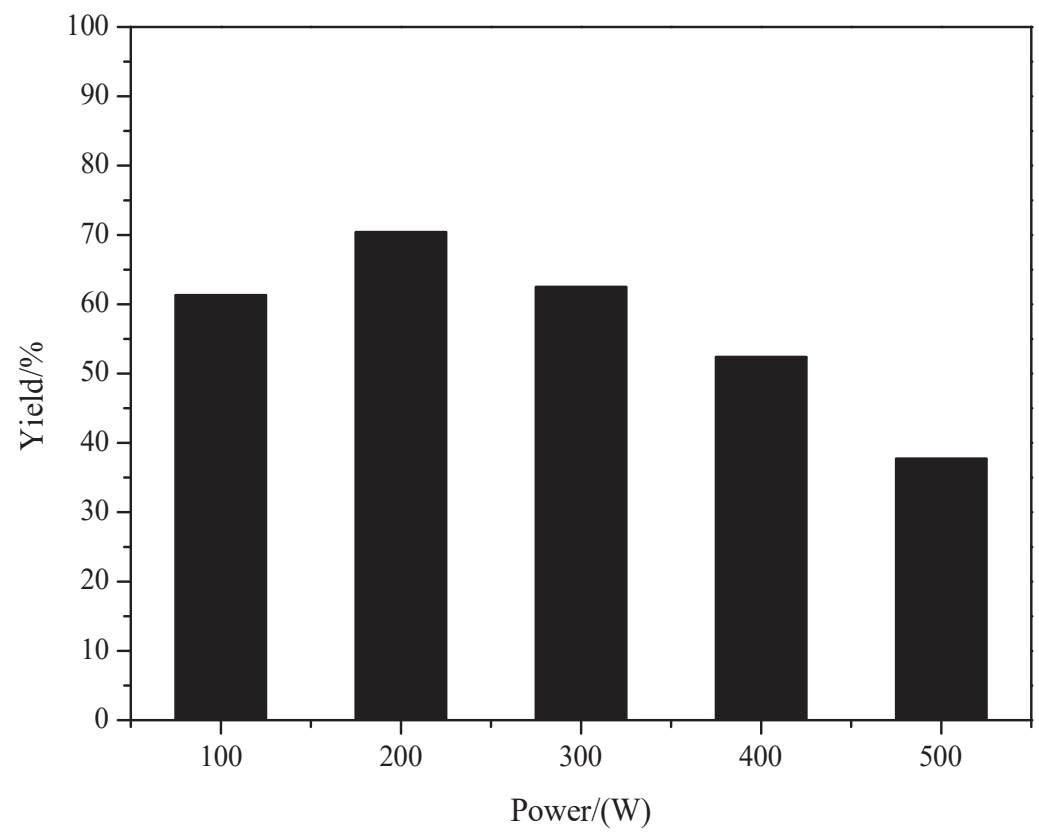

Figure 3. The effect of microwave irradiation power on compound 4i production.

In order to check the effect of the solvent on the reaction, various solvents were evaluated (Table 2). From an economical and environmental point of view, water was first screened as the reaction medium. But due to solubility problem, the yield of the reaction was very low (Table 2, entry 1). Next, organic solvents were screened as the reaction medium (Table 2, entries 2-5). It was found that the yield of compound $4 \mathbf{i}$ was much higher with ethyl alcohol as the solvent. Ethanol is relatively cheap, and environmentally friendly compared with other organic solvents.

Table 2. Effect of different solvents on the yield of $\mathbf{4 i}$

\begin{tabular}{ccccc}
\hline Entry & Solvent & Catalyst & Time $/$ min & Yield/\% \\
\hline 1 & $\mathrm{H}_{2} \mathrm{O}$ & $\mathrm{NH}_{4} \mathrm{OAc}$ & 5 & Trace \\
2 & $\mathrm{MeOH}$ & $\mathrm{NH}_{4} \mathrm{OAc}$ & 5 & 23 \\
3 & $\mathrm{CH}_{3} \mathrm{CN}$ & $\mathrm{NH}_{4} \mathrm{OAc}$ & 5 & 11 \\
4 & $\mathrm{DMF}$ & $\mathrm{NH}_{4} \mathrm{OAc}$ & 5 & 20 \\
5 & $\mathrm{EtOH}$ & $\mathrm{NH}_{4} \mathrm{OAc}$ & 5 & 70 \\
\hline
\end{tabular}




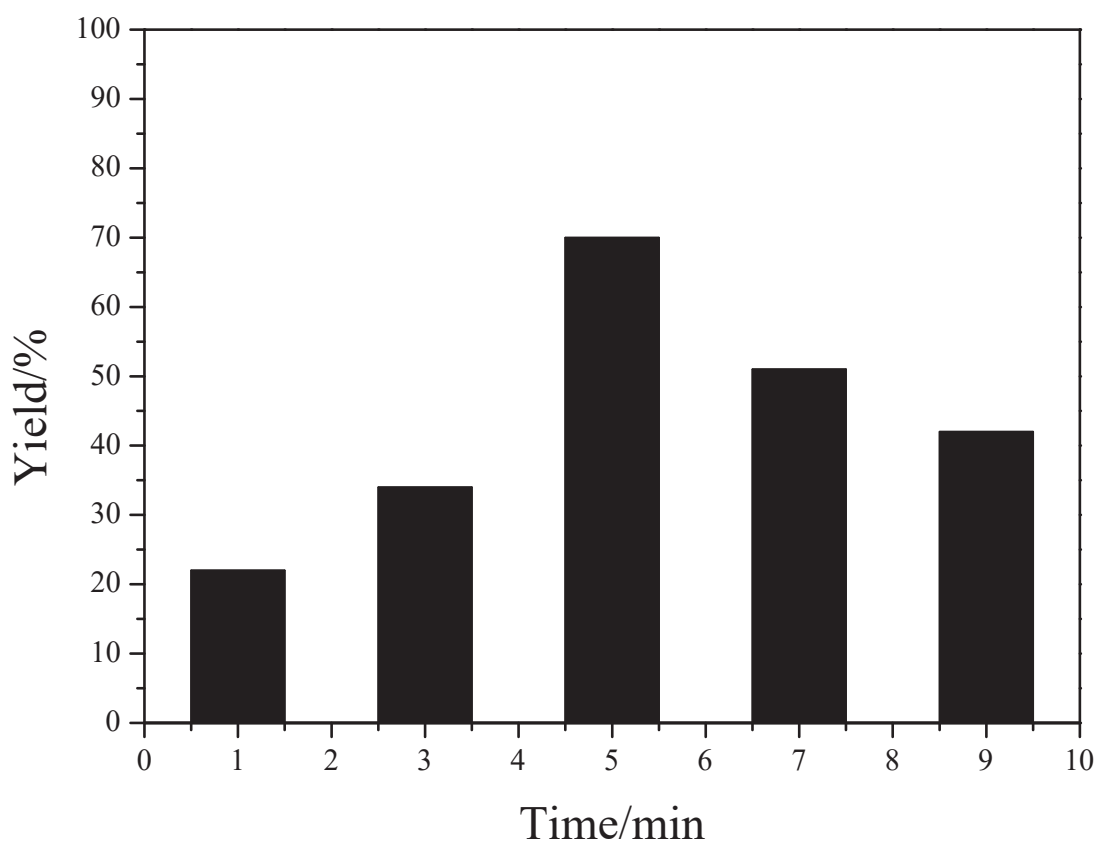

Figure 4. Change of yield of compound $4 \mathbf{i}$ over different microwave irradiation times.

When microwave irradiation is employed, the irradiation time is another important parameter in reaction optimization. Figure 4 indicates the interaction between the yield of product $4 \mathbf{i}$ and the microwave irradiation time. We tested the effects of five different reaction times on the yield. As shown in the Figure 4, the yield of $\mathbf{4 i}$ was speedily improved as the microwave irradiation time increased in the range from $1 \mathrm{~min}$ to $5 \mathrm{~min}$, because microwave irradiation can cause molecules of the reactants to vibrate and so bring the reaction temperature to its optimal value in a short time. But when the irradiation time exceeded 5 min the yield of compound $4 \mathbf{i}$ decreased, either because by-products were formed or the product $\mathbf{4 i}$ was decomposed. So, the ideal microwave irradiation time under our conditions was $5 \mathrm{~min}$.

With this result in hand, we went on to widen the scope of the methodology. Using the optimized reaction conditions, 4-hydroxycoumarin (5) was examined as a replacement for the 2pyrone. The reactions proceeded well and gave good yields (Table 3, entries 15-18). The results exhibit the scope and generality of the new three component cyclization with respect to a wide range of enol ketones and aldehyde substrates. As shown in Table 3, the yields of the reaction were much higher and the reaction times were much less when compared with conventional heating. These results further indicated that the microwave irradiation synthesis is a very effective method of building heterocyclic structures. The structures of all new compounds $\mathbf{4 a \sim 4 n}$ and $\mathbf{6 a} \sim \mathbf{6 d}$ were fully confirmed by means of IR, ${ }^{1} \mathrm{H}$ NMR, ${ }^{13} \mathrm{C}$ NMR and HRMS measurements . 
In order to know how the microwave irradiation promotes the reaction, taking into consideration the entire outcome, a possible reaction mechanism for the domino coupling is depicted in Scheme 2.

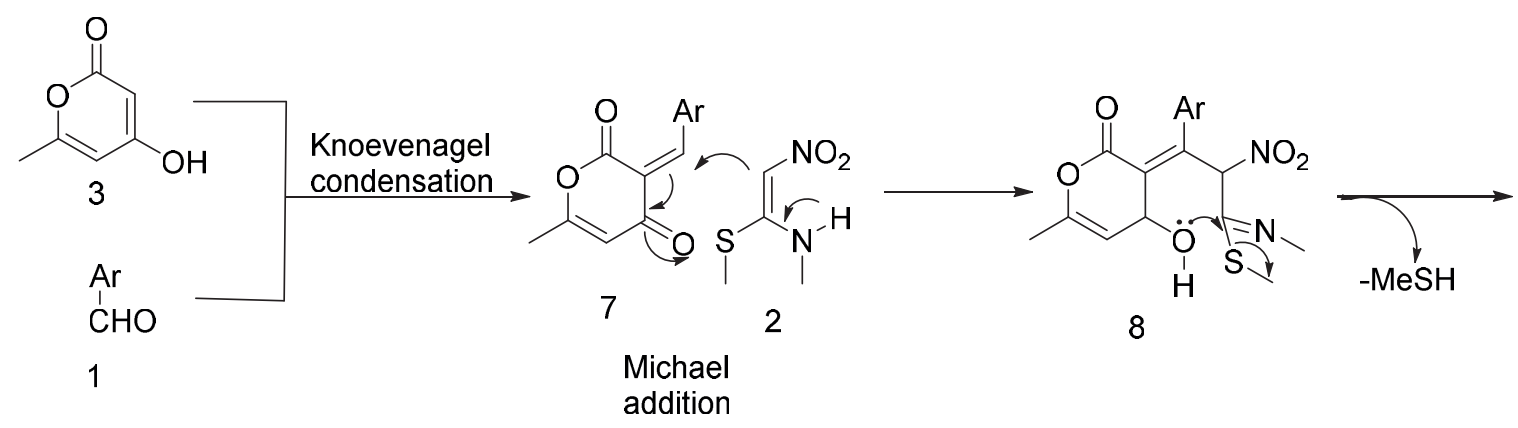<smiles>C=NC1OC2C=C(C)OC(=O)C2=C(Br)C1[N+](=O)[O-]</smiles><smiles>CNC1=C([N+](=O)[O-])C(Br)c2c(cc(C)oc2=O)O1</smiles>

Scheme 2. Possible reaction mechanism for the compounds 4.

The first step is the Knoevenagel condensation between compound $\mathbf{3}$ and aldehyde $\mathbf{1}$ that leads to the adduct 7, which acts as a Michael acceptor. The adduct 7 immediately undergoes Michael-type addition with NMSM 2 to generate the open-chain intermediate 8 . The intermediate $\mathbf{8}$ undergoes intramolecular O-cyclization to give compound $\mathbf{4}$ with elimination of $\mathrm{MeSH}$. 
Table 3. Three-component domino synthesis of products $\mathbf{4}$ and $\mathbf{6}$ under MW irradiation<smiles>CNC1=C([N+](=O)[O-])C(Br)c2c(cc(C)oc2=O)O1</smiles><smiles>CNC1=C([N+](=O)[O-])C(Br)c2c(c3ccccc3oc2=O)O1</smiles>

\begin{tabular}{|c|c|c|c|c|c|}
\hline \multirow{2}{*}{ Entry } & \multirow{2}{*}{$\mathrm{Ar}$} & \multicolumn{2}{|c|}{ Time } & \multicolumn{2}{|c|}{ Yield $(\%)$} \\
\hline & & $\mathrm{MW} / \mathrm{min}$ & $\mathrm{CH}^{\mathrm{a}} / \mathrm{h}$ & MW & $\mathrm{CH}^{\mathrm{a}}$ \\
\hline $4 a$ & $4-\mathrm{ClC}_{6} \mathrm{H}_{4}$ & 5 & 3 & 64 & 20 \\
\hline $4 b$ & $2-\mathrm{CH}_{3} \mathrm{OC}_{6} \mathrm{H}_{4}$ & 5 & 3 & 64 & 23 \\
\hline $4 c$ & $3-\mathrm{CH}_{3} \mathrm{C}_{6} \mathrm{H}_{4}$ & 5 & 3 & 70 & 24 \\
\hline $4 d$ & $\mathrm{C}_{6} \mathrm{H}_{5}$ & 5 & 3 & 69 & 24 \\
\hline $4 \mathrm{e}$ & $4-\mathrm{FC}_{6} \mathrm{H}_{4}$ & 5 & 3 & 56 & 21 \\
\hline $4 f$ & $3-\mathrm{FC}_{6} \mathrm{H}_{4}$ & 5 & 3 & 72 & 21 \\
\hline $4 \mathrm{~g}$ & $3,4-\mathrm{F}_{2} \mathrm{C}_{6} \mathrm{H}_{4}$ & 5 & 3 & 76 & 22 \\
\hline $4 \mathrm{~h}$ & $2-\mathrm{FC}_{6} \mathrm{H}_{4}$ & 5 & 3 & 70 & 32 \\
\hline $4 \mathrm{i}$ & $4-\mathrm{CH}_{3} \mathrm{OC}_{6} \mathrm{H}_{4}$ & 5 & 3 & 70. & 52 \\
\hline $4 j$ & 4- $\mathrm{CH}_{3} \mathrm{C}_{6} \mathrm{H}_{4}$ & 5 & 3 & 65 & 30 \\
\hline $4 \mathrm{k}$ & $2,4-\mathrm{Cl}_{2} \mathrm{C}_{6} \mathrm{H}_{3}$ & 5 & 3 & 73 & 33 \\
\hline 41 & $1-\mathrm{C}_{10} \mathrm{H}_{7}$ & 5 & 3 & 70 & 24 \\
\hline $4 m$ & $2-\mathrm{C}_{4} \mathrm{H}_{3} \mathrm{~S}$ & 5 & 3 & 76 & 41 \\
\hline $4 n$ & $2-\mathrm{C}_{4} \mathrm{H}_{3} \mathrm{O}$ & 5 & 3 & 76 & 34 \\
\hline $6 a$ & $2-\mathrm{OCH}_{3} \mathrm{C}_{6} \mathrm{H}_{4}$ & 5 & 3 & 69 & 27 \\
\hline $6 \mathrm{~b}$ & $4-\left(\mathrm{CH}_{3}\right)_{2} \mathrm{NC}_{6} \mathrm{H}_{4}$ & 5 & 3 & 74 & 32 \\
\hline $6 c$ & $2-\mathrm{C}_{4} \mathrm{H}_{3} \mathrm{~S}$ & 5 & 3 & 75 & 23 \\
\hline $6 \mathrm{~d}$ & $2-\mathrm{C}_{4} \mathrm{H}_{3} \mathrm{O}$ & 5 & 3 & 82 & 28 \\
\hline
\end{tabular}

${ }^{a}$ Conventional heating. ${ }^{b}$ Isolated yield. 


\section{Conclusions}

In summary, we have developed a three-component domino reaction [aldehydes, 4-hydroxy-6methyl-2-pyrone and $\mathrm{N}$-methyl-1-(methylthio)-2-nitroethenamine] for regioselective synthesis pyrano[4,3-b]pyran-5-one derivatives. The products 4 were obtained in good yields by selective $[1+3+2]$ cycloaddition in this work. The mild conditions, the maximum efficiency of a process and short reaction periods as well as operational simplicity are clearly represented in this one-pot transformation that provides an elegant methodology for the synthesis of highly functionalized pyrano[4,3-b]pyran-5-one derivatives. The present protocol is also extendable to a wide variety of substrates.

\section{Experimental Section}

General. ${ }^{1} \mathrm{H}$ and ${ }^{13} \mathrm{C}$ NMR spectra were recorded at 400 and $100 \mathrm{MHz}$ respectively on a Bruker AC-400 Avance spectrometer with DMSO- $d_{6}$ as solvent and TMS as internal standard; J values were given in Hertz (Hz). IR spectra were recorded on a Thermo iS5 FT instrument (KBr pellet). High resolution mass spectra were operated on Aglient 6550 iFunnel Q-TOF. A single-mode Microwave reactor (MAS-II) was used for microwave-mediated reactions. Melting points were measured on X-4 digital display melting point meter. All of the reagents were purchased from chemical reagent Crop. in China and used without further purification.

General synthetic procedure (conventional heating), exemplified by 7-methyl-2-methylamino-3-nitro-4-aryl-4H-pyrano[4,3-b]pyran-5-one (4i). Aromatic aldehyde $1 \mathrm{i}(0.5 \mathrm{mmol}$, $0.054 \mathrm{~g}, 1.0$ equiv), 4-hydroxy-6-methyl-2-pyrone 3 ( $0.5 \mathrm{mmol}, 0.063 \mathrm{~g}, 1.0$ equiv), NMSM 2 ( $0.5 \mathrm{mmol}, 0.074 \mathrm{~g}, 1.0$ equiv), and ammonium acetate $(0.5 \mathrm{mmol}, 0.039 \mathrm{~g}, 1.0$ equiv) as catalyst were added in a $25 \mathrm{~mL}$ three-necked flask. Then ethyl alcohol $(2 \mathrm{~mL})$ was added as the solvent. The resulting mixture was refluxed at $80{ }^{\circ} \mathrm{C}$ for $3 \mathrm{~h}$. During this time, a precipitate formed. After completion of the reaction (confirmed by TLC), the reaction mixture was cooled to room temperature. Then the precipitate was removed by filtration and washed with a little cold ethanol to give the pure product.

General procedure (microwave heating), exemplified by 7-methyl-2-methylamino-3-nitro4-aryl-4H-pyrano[4,3-b]pyran-5-one (4i). Aromatic aldehyde $1 \mathrm{i}(0.5 \mathrm{mmol}, 0.054 \mathrm{~g}, 1.0$ equiv), 4-hydroxy-6-methyl-2-pyrone 3 ( $0.5 \mathrm{mmol}, 0.063 \mathrm{~g}, 1.0$ equiv), NMSM 2 (0.5 mmol, $0.074 \mathrm{~g}, 1.0$ equiv) and ammonium acetate $(0.5 \mathrm{mmol}, 0.039 \mathrm{~g}, 1.0$ equiv) were mixed in ethyl alcohol $(2 \mathrm{~mL})$. The resulting mixture was heated at $80{ }^{\circ} \mathrm{C}$ under microwave irradiation $(200 \mathrm{~W})$ for $5 \mathrm{~min}$. After completion of the reaction (confirmed by TLC), the reaction mixture was cooled to room temperature. The solid was collected and washed with a little cold ethanol to give the pure product. 
4-(4-Chlorophenyl)-7-methyl-2-(methylamino)-3-nitro-4H-pyrano[4,3-b]pyran-5-one (4a). White powder; Yield; 64\%; mp 244-246 ${ }^{\circ}$; IR (KBr, v, $\left.\mathrm{cm}^{-1}\right)$ : 3222, 3083, 1727, 1670, 1640, $1600,1488,1442,1382,1360,1266,1145,1070,829,771,683 \mathrm{~cm}^{-1} ;{ }^{1} \mathrm{H}$ NMR $(400 \mathrm{MHz}$, DMSO- $\left.d_{6}\right): \delta_{\mathrm{H}} 2.24\left(3 \mathrm{H}, \mathrm{s}, \mathrm{O}-\mathrm{C}-\mathrm{CH}_{3}\right), 3.14\left(3 \mathrm{H}, \mathrm{d},{ }^{3} \mathrm{~J}_{\mathrm{HH}} 4.8 \mathrm{~Hz}, \mathrm{NH}-\mathrm{CH}_{3}\right), 4.90(1 \mathrm{H}, \mathrm{s}, \mathrm{C}-\mathrm{CH}-$ C), $6.56(1 \mathrm{H}, \mathrm{s}, \mathrm{C}=\mathrm{CH}-\mathrm{C}), 7.28-7.34(4 \mathrm{H}, \mathrm{m}, \mathrm{Ph}), 10.34\left(1 \mathrm{H}, \mathrm{q},{ }^{3} J_{\mathrm{HH}} 4.8 \mathrm{~Hz}, \mathrm{NH}\right) ;{ }^{13} \mathrm{C} \mathrm{NMR}$

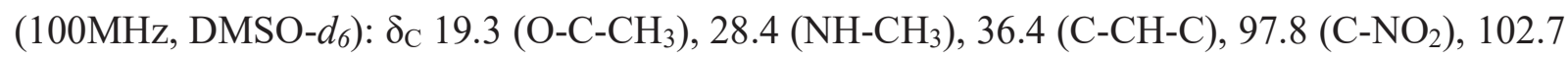
$(\mathrm{C}=\mathrm{C}-\mathrm{O}), 107.4(\mathrm{C}=\mathrm{C}-\mathrm{C}), 127.9$ (2C, benzene), 130.2 (2C, benzene), 131.4 (C-Cl, benzene), 140.5 (1C, benzene), $156.6(\mathrm{C}=\mathrm{C}-\mathrm{NH}), 156.9\left(\mathrm{CH}_{3}-\mathrm{C}-\mathrm{O}\right), 161.0(\mathrm{C}=\mathrm{O}), 163.3(\mathrm{C}=\mathrm{C}-\mathrm{O})$; HRMS (ESI) $m / z$ : calc. for: $\mathrm{C}_{16} \mathrm{H}_{13} \mathrm{~N}_{2} \mathrm{O}_{5} \mathrm{ClNa}, 371.0405[\mathrm{M}+\mathrm{Na}]^{+}$; found: 371.0413 .

\section{4-(2-Methoxyphenyl)-7-methyl-2-(methylamino)-3-nitro-4H-pyrano[4,3-b]pyran-5-one}

(4b). White powder; Yield; 64\%; mp 243-245 $\mathrm{C}^{\circ}$; IR (KBr, v, $\left.\mathrm{cm}^{-1}\right): 3229,3096,1718,1673$, 1642, 1600, 1448, 1381, 1357, 1265, 1186, 1136, 1059, 858, 808, $749 \mathrm{~cm}^{-1} ;{ }^{1} \mathrm{H}$ NMR $(400 \mathrm{MHz}$, DMSO- $\left.d_{6}\right): \delta_{\mathrm{H}} 2.23\left(3 \mathrm{H}, \mathrm{s}, \mathrm{O}-\mathrm{C}-\mathrm{CH}_{3}\right), 3.16\left(3 \mathrm{H}, \mathrm{d},{ }^{3} \mathrm{~J}_{\mathrm{HH}} 4.8 \mathrm{~Hz}, \mathrm{NH}-\mathrm{CH}_{3}\right), 3.64\left(3 \mathrm{H}, \mathrm{s}, \mathrm{O}-\mathrm{CH}_{3}\right)$, 4.90(1H, s, C-CH-C), 6.50 (1H, s, C=CH-C), 6.86-6.92 (2H, m, Ph), 7.17-7.21 (1H, m, Ph), 7.32$7.34(1 \mathrm{H}, \mathrm{m}, \mathrm{Ph}), 10.39\left(1 \mathrm{H}, \mathrm{d},{ }^{3} J_{\mathrm{HH}} 4.8 \mathrm{~Hz}, \mathrm{NH}\right) ;{ }^{13} \mathrm{C}$ NMR(100MHz,DMSO- $\left.d_{6}\right): \delta_{\mathrm{C}} 19.3(\mathrm{O}-\mathrm{C}-$ $\left.\mathrm{CH}_{3}\right), 28.2\left(\mathrm{NH}-\mathrm{CH}_{3}\right), 35.2(\mathrm{C}-\mathrm{CH}-\mathrm{C}), 55.4\left(\mathrm{O}-\mathrm{CH}_{3}\right), 97.6\left(\mathrm{C}-\mathrm{NO}_{2}\right), 101.2(\mathrm{C}=\mathrm{C}-\mathrm{O}), 106.7$ $(\mathrm{C}=\mathrm{C}-\mathrm{C}), 111.8,119.6,127.1,128.4$ and $132.8(5 \mathrm{C}$, benzene $), 157.0\left(1 \mathrm{C}, \mathrm{C}-\mathrm{OCH}_{3}\right), 157.5(\mathrm{C}=\mathrm{C}-$ $\mathrm{NH}), 157.9\left(\mathrm{CH}_{3}-\mathrm{C}-\mathrm{O}\right), 161.0 \quad(\mathrm{C}=\mathrm{O}), 162.8 \quad(\mathrm{C}=\mathrm{C}-\mathrm{O}) ; \mathrm{HRMS}$ (ESI) $m / z$ : calc. for: $\mathrm{C}_{17} \mathrm{H}_{16} \mathrm{~N}_{2} \mathrm{O}_{6} \mathrm{Na}, 367.0901[\mathrm{M}+\mathrm{Na}]^{+}$; found: 367.0897 .

7-Methyl-2-(methylamino)-3-nitro-4-m-tolyl-4H-pyrano[4,3-b]pyran-5-one (4c). White powder; Yield; 70\%; mp 225-227 ${ }^{\circ}$; IR (KBr, v, $\left.\mathrm{cm}^{-1}\right)$ : 3212, 3098, 1729, 1672, 1640, 1600, 1445, 1381, 1358, 1264, 1192, 1136,1062, 808, 765, $692 \mathrm{~cm}^{-1},{ }^{1} \mathrm{H}$ NMR (400 MHz, DMSO-d6): $\delta_{\mathrm{H}} 2.24\left(3 \mathrm{H}, \mathrm{s}, \mathrm{O}-\mathrm{C}-\mathrm{CH}_{3}\right), 2.26\left(3 \mathrm{H}, \mathrm{s}, \mathrm{CH}_{3}\right), 3.15\left(3 \mathrm{H}, \mathrm{d},{ }^{3} J_{\mathrm{HH}} 4.8 \mathrm{~Hz}, \mathrm{NH}-\mathrm{CH}_{3}\right), 4.90(1 \mathrm{H}, \mathrm{s}, \mathrm{C}-$ CH-C), $6.55(1 \mathrm{H}, \mathrm{s}, \mathrm{C}=\mathrm{CH}-\mathrm{C}), 6.99-7.10(3 \mathrm{H}, \mathrm{m}, \mathrm{Ph}), 7.13-7.16(1 \mathrm{H}, \mathrm{m}, \mathrm{Ph}), 10.32\left(1 \mathrm{H}, \mathrm{d},{ }^{3} \mathrm{JHH}_{\mathrm{HH}}\right.$ $5.2 \mathrm{~Hz}, \mathrm{NH}) ;{ }^{13} \mathrm{C} \mathrm{NMR}\left(100 \mathrm{MHz}, \mathrm{DMSO}-d_{6}\right): \delta_{\mathrm{C}} 19.3\left(\mathrm{O}-\mathrm{C}-\mathrm{CH}_{3}\right), 21.0(\mathrm{C}-\mathrm{CH} 3), 28.4(\mathrm{NH}-$ $\left.\mathrm{CH}_{3}\right), 36.6(\mathrm{C}-\mathrm{CH}-\mathrm{C}), 97.8\left(\mathrm{C}-\mathrm{NO}_{2}\right), 103.4(\mathrm{C}=\mathrm{C}-\mathrm{O}), 107.8(\mathrm{C}=\mathrm{C}-\mathrm{C}), 125.4,127.6,127.9,128.6$, 137.0 and $141.5\left(6 \mathrm{C}\right.$, benzene), $156.5(\mathrm{C}=\mathrm{C}-\mathrm{NH}), 157.0\left(\mathrm{CH}_{3}-\mathrm{C}-\mathrm{O}\right), 161.1(\mathrm{C}=\mathrm{O}), 163.0(\mathrm{C}=\mathrm{C}-$ O); HRMS (ESI) $m / z$ : calc. for: $\mathrm{C}_{17} \mathrm{H}_{16} \mathrm{~N}_{2} \mathrm{O}_{5} \mathrm{Na}, 351.0951[\mathrm{M}+\mathrm{Na}]^{+}$; found: 351.0947 .

7-Methyl-2-(methylamino)-3-nitro-4-phenyl-4H-pyrano[4,3-b]pyran-5-one (4d). White powder; Yield; 69\%; mp 244-247º $\mathrm{C}$; IR (KBr, v, $\left.\mathrm{cm}^{-1}\right): 3208,3067,1733,1676,1638,1606$, $1454,1389,1359,1264,1188,1139,1066,838,757,702 \mathrm{~cm}^{-1} ;{ }^{1} \mathrm{H}$ NMR (400 MHz, DMSO- $\left.d_{6}\right)$ : $\delta_{\mathrm{H}} 2.24\left(3 \mathrm{H}, \mathrm{s}, \mathrm{O}-\mathrm{C}-\mathrm{CH}_{3}\right), 3.15\left(3 \mathrm{H}, \mathrm{s}, \mathrm{NH}-\mathrm{CH}_{3}\right), 4.92(1 \mathrm{H}, \mathrm{s}, \mathrm{C}-\mathrm{CH}-\mathrm{C}), 6.56(1 \mathrm{H}, \mathrm{s}, \mathrm{C}=\mathrm{CH}-\mathrm{C})$, 7.17-7.27 (5H, m, Ph), $10.34(1 \mathrm{H}, \mathrm{s}, \mathrm{NH}) ;{ }^{13} \mathrm{C}$ NMR (100MHz, DMSO- $\left.d_{6}\right): \delta_{\mathrm{C}} 19.3\left(\mathrm{O}-\mathrm{C}-\mathrm{CH}_{3}\right)$, $28.4\left(\mathrm{NH}_{-} \mathrm{CH}_{3}\right), 36.7(\mathrm{C}-\mathrm{CH}-\mathrm{C}), 97.8\left(\mathrm{C}-\mathrm{NO}_{2}\right), 103.3(\mathrm{C}=\mathrm{C}-\mathrm{O}), 107.8(\mathrm{C}=\mathrm{C}-\mathrm{C}), 126.9,128.0$ (2C), $128.2(2 \mathrm{C})$ and $141.6\left(6 \mathrm{C}\right.$, benzene), $156.6(\mathrm{C}=\mathrm{C}-\mathrm{NH}), 157.0\left(\mathrm{CH}_{3}-\mathrm{C}-\mathrm{O}\right), 161.0(\mathrm{C}=\mathrm{O})$, $163.1(\mathrm{C}=\mathrm{C}-\mathrm{O})$; HRMS (ESI) $m / z$ : calc. for: $\mathrm{C}_{16} \mathrm{H}_{14} \mathrm{~N}_{2} \mathrm{O}_{5} \mathrm{Na}, 337.0795[\mathrm{M}+\mathrm{Na}]^{+}$; found: 337.0800 .

4-(4-Fluorophenyl)-7-methyl-2-(methylamino)-3-nitro-4H-pyrano[4,3-b]pyran-5-one (4e). White powder; Yield; 56\%; mp 234-236 ${ }^{\circ} \mathrm{C}$; IR (KBr, v, $\left.\mathrm{cm}^{-1}\right): 3226,3085,1729,1671,1641$, $1600,1505,1431,1383,1361,1222,1145,1070,830,773,740 \mathrm{~cm}^{-1} ;^{1} \mathrm{H}$ NMR (400 MHz, 
DMSO- $\left.d_{6}\right): \delta_{\mathrm{H}} 2.13\left(3 \mathrm{H}, \mathrm{s}, \mathrm{O}-\mathrm{C}-\mathrm{CH}_{3}\right), 3.03\left(3 \mathrm{H}, \mathrm{d},{ }^{3} \mathrm{~J}_{\mathrm{HH}} 5.2 \mathrm{~Hz}, \mathrm{NH}-\mathrm{CH}_{3}\right), 4.80(1 \mathrm{H}, \mathrm{s}, \mathrm{C}-\mathrm{CH}-$ C), $6.45(1 \mathrm{H}, \mathrm{s}, \mathrm{C}=\mathrm{CH}-\mathrm{C}), 6.95-6.99(2 \mathrm{H}, \mathrm{m}, \mathrm{Ph}), 7.18-7.22(2 \mathrm{H}, \mathrm{m}, \mathrm{Ph}), 10.24\left(1 \mathrm{H}, \mathrm{d},{ }^{3} J_{\mathrm{HH}} 5.2\right.$ $\mathrm{Hz}, \mathrm{NH}) ;{ }^{13} \mathrm{C}$ NMR $\left(100 \mathrm{MHz}, \mathrm{DMSO}-d_{6}\right): \delta_{\mathrm{C}} 19.3\left(\mathrm{O}-\mathrm{C}-\mathrm{CH}_{3}\right), 28.4\left(\mathrm{NH}-\mathrm{CH}_{3}\right), 36.1(\mathrm{C}-\mathrm{CH}-\mathrm{C})$, $97.8\left(\mathrm{C}-\mathrm{NO}_{2}\right), 103.0(\mathrm{C}=\mathrm{C}-\mathrm{O}), 107.6(\mathrm{C}=\mathrm{C}-\mathrm{C}), 114.6,130.1(2 \mathrm{C}), 137.7(2 \mathrm{C})$ and $156.7(6 \mathrm{C}$, benzene), $159.8(\mathrm{C}=\mathrm{C}-\mathrm{NH}), 161.0\left(\mathrm{CH}_{3}-\mathrm{C}-\mathrm{O}\right), 162.2(\mathrm{C}=\mathrm{O}), 163.2(\mathrm{C}=\mathrm{C}-\mathrm{O})$; HRMS (ESI) $\mathrm{m} / z$ : calc. for: $\mathrm{C}_{16} \mathrm{H}_{13} \mathrm{~N}_{2} \mathrm{O}_{5} \mathrm{FNa}, 355.0701[\mathrm{M}+\mathrm{Na}]^{+}$; found: 355.0708 .

4-(3-Fluorophenyl)-7-methyl-2-(methylamino)-3-nitro-4H-pyrano[4,3-b]pyran-5-one(4f).

White powder; Yield; 72\%; mp 226-228 ${ }^{\circ} \mathrm{C}$; IR (KBr, $\left.v, \mathrm{~cm}^{-1}\right): 3197,3088,1725,1675,1634$, 1603, 1448, 1389, 1351, 1263, 1189, 1136, 1061, 808, 789, $689 \mathrm{~cm}^{-1} ;{ }_{1}^{1} \mathrm{H}$ NMR (400 MHz, DMSO- $\left.d_{6}\right): \delta_{\mathrm{H}} 2.24\left(3 \mathrm{H}, \mathrm{s}, \mathrm{O}-\mathrm{C}-\mathrm{CH}_{3}\right), 3.14\left(3 \mathrm{H}, \mathrm{d},{ }^{3} \mathrm{~J}_{\mathrm{HH}} 4.4 \mathrm{~Hz}, \mathrm{NH}-\mathrm{CH}_{3}\right), 4.93(1 \mathrm{H}, \mathrm{s}, \mathrm{C}-\mathrm{CH}-$ C), $6.56(1 \mathrm{H}, \mathrm{s}, \mathrm{C}=\mathrm{CH}-\mathrm{C}), 7.01-7.13(3 \mathrm{H}, \mathrm{m}, \mathrm{Ph}), 7.28-7.33(1 \mathrm{H}, \mathrm{m}, \mathrm{Ph}), 10.37\left(1 \mathrm{H}, \mathrm{d},{ }^{3} J_{\mathrm{HH}} 4.4\right.$ $\mathrm{Hz}, \mathrm{NH}) ;{ }^{13} \mathrm{C}$ NMR $\left(100 \mathrm{MHz}, \mathrm{DMSO}-d_{6}\right): \delta_{\mathrm{C}} 19.3\left(\mathrm{O}-\mathrm{C}-\mathrm{CH}_{3}\right), 28.5\left(\mathrm{NH}-\mathrm{CH}_{3}\right), 36.7(\mathrm{C}-\mathrm{CH}-\mathrm{C})$, $97.8\left(\mathrm{C}-\mathrm{NO}_{2}\right), 102.6(\mathrm{C}=\mathrm{C}-\mathrm{O}), 107.3(\mathrm{C}=\mathrm{C}-\mathrm{C}), 113.7,115.2,124.3,129.8,144.3,156.8(6 \mathrm{C}$, benzene), $160.6(\mathrm{C}=\mathrm{C}-\mathrm{NH}), 161.0\left(\mathrm{CH}_{3}-\mathrm{C}-\mathrm{O}\right), 163.0(\mathrm{C}=\mathrm{O}), 163.3(\mathrm{C}=\mathrm{C}-\mathrm{O})$; HRMS (ESI) $m / z$ : calc. for: $\mathrm{C}_{16} \mathrm{H}_{13} \mathrm{~N}_{2} \mathrm{O}_{5} \mathrm{FNa}, 355.0701[\mathrm{M}+\mathrm{Na}]^{+}$; found: 355.0706 .

4-(3,4-Difluorophenyl)-7-methyl-2-(methylamino)-3-nitro-4H-pyrano[4,3-b]pyran-5-one

(4g). White powder; Yield; 76\%; mp 192-194 ${ }^{\circ} \mathrm{C}$; IR (KBr, v, $\left.\mathrm{cm}^{-1}\right)$ : 3223, 3087, 1727, 1671, 1640, 1599, 1514, 1434, 1383, 1363, 1267, 1145, 1072, 829, 768, $668 \mathrm{~cm}^{-1} ;{ }^{1} \mathrm{H}$ NMR (400 MHz, DMSO-d $)_{6}: \delta_{\mathrm{H}} 2.24\left(3 \mathrm{H}, \mathrm{s}, \mathrm{O}-\mathrm{C}-\mathrm{CH}_{3}\right), 3.14\left(3 \mathrm{H}, \mathrm{d},{ }^{3} \mathrm{~J}_{\mathrm{HH}} 4.8 \mathrm{~Hz}, \mathrm{NH}-\mathrm{CH}_{3}\right), 4.91(1 \mathrm{H}, \mathrm{s}, \mathrm{C}-\mathrm{CH}-$ C), $6.56(1 \mathrm{H}, \mathrm{s}, \mathrm{C}=\mathrm{CH}-\mathrm{C}), 7.14-7.16(1 \mathrm{H}, \mathrm{m}, \mathrm{Ph}), 7.28-7.38(2 \mathrm{H}, \mathrm{m}, \mathrm{Ph}), 10.37\left(1 \mathrm{H}, \mathrm{d},{ }^{3} J_{\mathrm{HH}} 4.8\right.$ $\mathrm{Hz}, \mathrm{NH}) ;{ }^{13} \mathrm{C}$ NMR $\left(100 \mathrm{MHz}, \mathrm{DMSO}-d_{6}\right): \delta_{\mathrm{C}} 19.3\left(\mathrm{O}-\mathrm{C}-\mathrm{CH}_{3}\right), 28.4\left(\mathrm{NH}-\mathrm{CH}_{3}\right), 36.4(\mathrm{C}-\mathrm{CH}-\mathrm{C})$, $97.8\left(\mathrm{C}-\mathrm{NO}_{2}\right), 102.3(\mathrm{C}=\mathrm{C}-\mathrm{O}), 107.2(\mathrm{C}=\mathrm{C}-\mathrm{C}), 116.7,117.3,125.1,139.2,147.4$ and, $149.9(6 \mathrm{C}$, benzene), $156.7(\mathrm{C}=\mathrm{C}-\mathrm{NH}), 156.9\left(\mathrm{CH}_{3}-\mathrm{C}-\mathrm{O}\right), 161.0(\mathrm{C}=\mathrm{O}), 163.4(\mathrm{C}=\mathrm{C}-\mathrm{O})$; HRMS (ESI) $m / z$ : calc. for: $\mathrm{C}_{16} \mathrm{H}_{12} \mathrm{~N}_{2} \mathrm{O}_{5} \mathrm{~F}_{2} \mathrm{Na}, 373.0606[\mathrm{M}+\mathrm{Na}]^{+}$; found: 373.0611 .

4-(2-Fluorophenyl)-7-methyl-2-(methylamino)-3-nitro-4H-pyrano[4,3-b]pyran-5-one(4h).

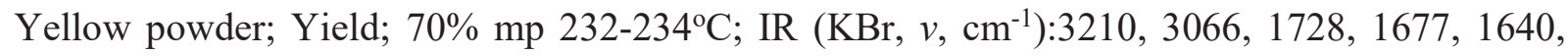
1604, 1492, 1391, 1361, 1265, 1190, 1143, 1067, 840, 782, $747 \mathrm{~cm}^{-1} ;{ }^{1} \mathrm{H}$ NMR (400 MHz, DMSO- $\left.d_{6}\right): \delta_{\mathrm{H}} 2.24\left(3 \mathrm{H}, \mathrm{s}, \mathrm{O}-\mathrm{C}-\mathrm{CH}_{3}\right), 3.14\left(3 \mathrm{H}, \mathrm{d},{ }^{3} \mathrm{~J}_{\mathrm{HH}} 4.8 \mathrm{~Hz}, \mathrm{NH}-\mathrm{CH}_{3}\right), 5.08(1 \mathrm{H}, \mathrm{s}, \mathrm{C}-\mathrm{CH}-$ C), $6.56(1 \mathrm{H}, \mathrm{s}, \mathrm{C}=\mathrm{CH}-\mathrm{C}), 7.05-7.13(2 \mathrm{H}, \mathrm{m}, \mathrm{Ph}), 7.22-7.27(1 \mathrm{H}, \mathrm{m}, \mathrm{Ph}), 7.33-7.37(1 \mathrm{H}, \mathrm{m}, \mathrm{Ph})$, $10.38\left(1 \mathrm{H}, \mathrm{d},{ }^{3} \mathrm{~J}_{\mathrm{HH}} 4.8 \mathrm{~Hz}, \mathrm{NH}\right) ;{ }^{13} \mathrm{C} \mathrm{NMR}\left(100 \mathrm{MHz}, \mathrm{DMSO}-d_{6}\right): \delta_{\mathrm{C}} 19.3\left(\mathrm{O}-\mathrm{C}-\mathrm{CH}_{3}\right), 28.6(\mathrm{NH}-$ $\left.\mathrm{CH}_{3}\right), 32.2$ (C-CH-C), $97.7\left(\mathrm{C}-\mathrm{NO}_{2}\right), 101.6(\mathrm{C}=\mathrm{C}-\mathrm{O}), 106.8(\mathrm{C}=\mathrm{C}-\mathrm{C}), 115.2,123.8,127.6,128.9$, 131.7 and $156.9\left(6 \mathrm{C}\right.$, benzene), $159.4(\mathrm{C}=\mathrm{C}-\mathrm{NH}), 160.9\left(\mathrm{CH}_{3}-\mathrm{C}-\mathrm{O}\right), 161.9(\mathrm{C}=\mathrm{O}), 163.3(\mathrm{C}=\mathrm{C}-$ O); HRMS (ESI) $m / z$ : calc. for: $\mathrm{C}_{16} \mathrm{H}_{13} \mathrm{~N}_{2} \mathrm{O}_{5} \mathrm{Na}, 355.0701[\mathrm{M}+\mathrm{Na}]^{+}$; found: 355.0711 .

4-(4-Methoxyphenyl)-7-methyl-2-(methylamino)-3-nitro-4H-pyrano[4,3-b]pyran-5-one(4i). White powder; Yield; 70\%; mp 238-240 ${ }^{\circ}$; IR (KBr, v, $\left.\mathrm{cm}^{-1}\right): 3219,3081,1728,1670,1640$, 1600, 1508, 1379, 1357, 1251, 1171, 1143, 1067, 831, 772, $708 \mathrm{~cm}^{-1} ;{ }^{1} \mathrm{H}$ NMR (400 MHz, DMSO- $\left.d_{6}\right): \delta_{\mathrm{H}} 2.23\left(3 \mathrm{H}, \mathrm{s}, \mathrm{O}-\mathrm{C}-\mathrm{CH}_{3}\right), 3.14\left(3 \mathrm{H}, \mathrm{d},{ }^{3} J_{\mathrm{HH}} 4.0 \mathrm{~Hz}, \mathrm{NH}-\mathrm{CH}_{3}\right), 3.70\left(3 \mathrm{H}, \mathrm{s}, \mathrm{OCH}_{3}\right)$, $4.86(1 \mathrm{H}, \mathrm{s}, \mathrm{C}-\mathrm{CH}-\mathrm{C}), 6.55(1 \mathrm{H}, \mathrm{s}, \mathrm{C}=\mathrm{CH}-\mathrm{C}), 6.79-6.83(2 \mathrm{H}, \mathrm{m}, \mathrm{Ph}), 7.14-7.18(2 \mathrm{H}, \mathrm{m}, \mathrm{Ph})$, $10.32\left(1 \mathrm{H}, \mathrm{d},{ }^{3} \mathrm{~J}_{\mathrm{HH}} 4.4 \mathrm{~Hz}, \mathrm{NH}\right) ;{ }^{13} \mathrm{C} \mathrm{NMR}\left(100 \mathrm{MHz}, \mathrm{DMSO}-d_{6}\right): \delta_{\mathrm{C}} 19.3\left(\mathrm{O}-\mathrm{C}-\mathrm{CH}_{3}\right), 28.4(\mathrm{NH}-$ $\left.\mathrm{CH}_{3}\right), 35.8$ (C-CH-C), $55.0\left(\mathrm{O}-\mathrm{CH}_{3}\right), 97.8\left(\mathrm{C}-\mathrm{NO}_{2}\right), 103.5(\mathrm{C}=\mathrm{C}-\mathrm{O}), 107.9(\mathrm{C}=\mathrm{C}-\mathrm{C}), 113.4$, 
$129.2(2 \mathrm{C}), 133.6(2 \mathrm{C})$ and $156.4\left(6 \mathrm{C}\right.$, benzene), $157.0(\mathrm{C}=\mathrm{C}-\mathrm{NH}), 158.1\left(\mathrm{CH}_{3}-\mathrm{C}-\mathrm{O}\right), 161.1$ $(\mathrm{C}=\mathrm{O}), 162.9(\mathrm{C}=\mathrm{C}-\mathrm{O})$; HRMS (ESI) $m / z$ : calc. for: $\mathrm{C}_{17} \mathrm{H}_{16} \mathrm{~N}_{2} \mathrm{O}_{6} \mathrm{Na}, 367.0901[\mathrm{M}+\mathrm{Na}]^{+}$; found: 367.0892 .

7-Methyl-2-(methylamino)-3-nitro-4-p-tolyl-4H-pyrano[4,3-b]pyran-5-one $\quad(4 \mathbf{j}) . \quad$ White powder; Yield; $65 \%$; mp 239-241 ${ }^{\circ} \mathrm{C}$; IR (KBr, v, $\left.\mathrm{cm}^{-1}\right)$ : 3223, 3083, 1731, 1670, 1640, 1599, 1381, 1360, 1266, 1245, 1144, 1069, 829, 770, 686, $668 \mathrm{~cm}^{-1},{ }_{1}^{1} \mathrm{H}$ NMR (400 MHz, DMSO- $\left.d_{6}\right)$ : $\delta_{\mathrm{H}} 2.23\left(6 \mathrm{H}, \mathrm{s},-\mathrm{CH}_{3}\right), 3.14\left(3 \mathrm{H}, \mathrm{d},{ }^{3} J_{\mathrm{HH}} 5.2 \mathrm{~Hz}, \mathrm{NH}-\mathrm{CH}_{3}\right), 4.88(1 \mathrm{H}, \mathrm{s}, \mathrm{C}-\mathrm{CH}-\mathrm{C}), 6.55(1 \mathrm{H}, \mathrm{s}$, $\mathrm{C}=\mathrm{CH}-\mathrm{C}), 7.06-7.13(4 \mathrm{H}, \mathrm{m}, \mathrm{Ph}), 10.32\left(1 \mathrm{H}, \mathrm{d},{ }^{3} J_{\mathrm{HH}} 5.2 \mathrm{~Hz}, \mathrm{NH}\right) ;{ }^{13} \mathrm{C}$ NMR $(100 \mathrm{MHz}, \mathrm{DMSO}-$

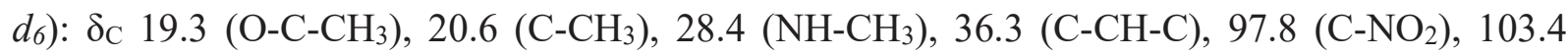
$(\mathrm{C}=\mathrm{C}-\mathrm{O}), 107.8(\mathrm{C}=\mathrm{C}-\mathrm{C}), 128.0,128.5(2 \mathrm{C}), 136.0(2 \mathrm{C})$, and $138.6(6 \mathrm{C}$, benzene $), 156.4(\mathrm{C}=\mathrm{C}-$ $\mathrm{NH}), \quad 157.0 \quad\left(\mathrm{CH}_{3}-\mathrm{C}-\mathrm{O}\right), 161.0 \quad(\mathrm{C}=\mathrm{O}), 162.9 \quad(\mathrm{C}=\mathrm{C}-\mathrm{O}) ; \mathrm{HRMS} \quad(\mathrm{ESI}) \mathrm{m} / z$ : calc. for: $\mathrm{C}_{17} \mathrm{H}_{16} \mathrm{~N}_{2} \mathrm{O}_{5} \mathrm{Na}, 351.0951[\mathrm{M}+\mathrm{Na}]^{+}$; found: 351.0943 .

4-(2,4-Dichlorophenyl)-7-methyl-2-(methylamino)-3-nitro-4H-pyrano[4,3-b]pyran-5-one

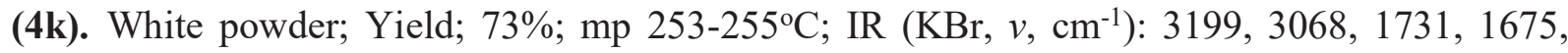
1639, 1603, 1471, 1445, 1390, 1361, 1264, 1140, 1067, 832, 731, $694 \mathrm{~cm}^{-1} ;{ }^{1} \mathrm{H}$ NMR $(400 \mathrm{MHz}$, DMSO- $\left.d_{6}\right): \delta_{\mathrm{H}} 2.24\left(3 \mathrm{H}, \mathrm{s}, \mathrm{O}-\mathrm{C}-\mathrm{CH}_{3}\right), 3.14\left(3 \mathrm{H}, \mathrm{d},{ }^{3} \mathrm{~J}_{\mathrm{HH}} 5.2 \mathrm{~Hz}, \mathrm{NH}-\mathrm{CH}_{3}\right), 5.21(1 \mathrm{H}, \mathrm{s}, \mathrm{C}-\mathrm{CH}-$ C), $6.55(1 \mathrm{H}, \mathrm{s}, \mathrm{C}=\mathrm{CH}-\mathrm{C}), 7.32-7.35(1 \mathrm{H}, \mathrm{m}, \mathrm{Ph}), 7.42-7.47(2 \mathrm{H}, \mathrm{m}, \mathrm{Ph}), 10.43\left(1 \mathrm{H}, \mathrm{d},{ }^{3} J_{\mathrm{HH}} 5.2\right.$ $\mathrm{Hz}, \mathrm{NH}) ;{ }^{13} \mathrm{C}$ NMR $\left(100 \mathrm{MHz}, \mathrm{DMSO}-d_{6}\right): \delta_{\mathrm{C}} 19.3\left(\mathrm{O}-\mathrm{C}-\mathrm{CH}_{3}\right), 28.4\left(\mathrm{NH}-\mathrm{CH}_{3}\right), 35.5(\mathrm{C}-\mathrm{CH}-\mathrm{C})$, $97.6\left(\mathrm{C}-\mathrm{NO}_{2}\right), 100.9(\mathrm{C}=\mathrm{C}-\mathrm{O}), 106.5(\mathrm{C}=\mathrm{C}-\mathrm{C}), 126.7,128.7,132.1,134.0,134.1$ and $136.9(6 \mathrm{C}$, benzene), $157.0(\mathrm{C}=\mathrm{C}-\mathrm{NH}), 160.7\left(2 \mathrm{C}, \mathrm{CH}_{3}-\mathrm{C}-\mathrm{O}, \mathrm{C}=\mathrm{O}\right), 163.6(\mathrm{C}=\mathrm{C}-\mathrm{O})$; HRMS (ESI) $\mathrm{m} / z$ : calc. for: $\mathrm{C}_{16} \mathrm{H}_{12} \mathrm{~N}_{2} \mathrm{O}_{5} \mathrm{Cl}_{2} \mathrm{Na}, 405.0015[\mathrm{M}+\mathrm{Na}]^{+}$; found: 405.0027.

7-Methyl-2-(methylamino)-4-naphthalen-1-yl-3-nitro-4H-pyrano[4,3-b]pyran-5-one

(41).

White powder; Yield; 70\%; mp 259-261 ${ }^{\circ} \mathrm{C}$; IR (KBr, $\left.v, \mathrm{~cm}^{-1}\right): 3192,3062,1731,1672,1640$, 1600, 1441, 1387, 1363, 1261, 1188, 1135, 1063, 799, 784, $694 \mathrm{~cm}^{-1} ;{ }^{1} \mathrm{H}$ NMR (400 MHz, DMSO- $\left.d_{6}\right): \delta_{\mathrm{H}} 2.22\left(3 \mathrm{H}, \mathrm{s}, \mathrm{O}-\mathrm{C}-\mathrm{CH}_{3}\right), 3.19\left(3 \mathrm{H}, \mathrm{d},{ }^{3} \mathrm{~J}_{\mathrm{HH}} 4.8 \mathrm{~Hz}, \mathrm{NH}-\mathrm{CH}_{3}\right), 5.85(1 \mathrm{H}, \mathrm{s}, \mathrm{C}-\mathrm{CH}-$ C), $6.59(1 \mathrm{H}, \mathrm{s}, \mathrm{C}=\mathrm{CH}-\mathrm{C}), 7.27-7.89(6 \mathrm{H}, \mathrm{m}, \mathrm{Ph}), 8.63\left(1 \mathrm{H}, \mathrm{d},{ }^{3} \mathrm{~J}_{\mathrm{HH}} 8.8 \mathrm{~Hz}, \mathrm{Ph}\right), 10.36(1 \mathrm{H}, \mathrm{d}$, $\left.{ }^{3} J_{\mathrm{HH}} 4.8 \mathrm{~Hz}, \mathrm{NH}\right) ;{ }^{13} \mathrm{C} \mathrm{NMR}\left(100 \mathrm{MHz}, \mathrm{DMSO}-d_{6}\right): \delta_{\mathrm{C}} 19.3\left(\mathrm{O}-\mathrm{C}-\mathrm{CH}_{3}\right), 28.5\left(\mathrm{NH}-\mathrm{CH}_{3}\right), 31.5(\mathrm{C}-$ $\mathrm{CH}-\mathrm{C}), 97.8\left(\mathrm{C}-\mathrm{NO}_{2}\right), 104.4(\mathrm{C}=\mathrm{C}-\mathrm{O}), 109.2(\mathrm{C}=\mathrm{C}-\mathrm{C}), 124.8,125.3,125.4,125.5,126.0,127.4$, 127.9, 131.8, 132.7 and 140.1 (10C, naphthalene), $156.4(\mathrm{C}=\mathrm{C}-\mathrm{NH}), 156.9\left(\mathrm{CH}_{3}-\mathrm{C}-\mathrm{O}\right), 161.1$ $(\mathrm{C}=\mathrm{O}), 162.7(\mathrm{C}=\mathrm{C}-\mathrm{O})$; HRMS (ESI) $m / z$ : calc. for: $\mathrm{C}_{20} \mathrm{H}_{16} \mathrm{~N}_{2} \mathrm{O}_{5} \mathrm{Na}, 387.0951[\mathrm{M}+\mathrm{Na}]^{+}$; found: 387.0947 .

7-Methyl-2-(methylamino)-3-nitro-4-thiophen-2-yl-4H-pyrano[4,3-b]pyran-5-one $\quad(4 \mathrm{~m})$. White powder; Yield; 76\%; mp 224-226 ${ }^{\circ}$; IR (KBr, v, $\left.\mathrm{cm}^{-1}\right): 3197,3069,1730,1674,1639$, 1604, 1439, 1389, 1358, 1257, 1189, 1140, 1066, 835, 725, $693 \mathrm{~cm}^{-1} ;{ }^{1} \mathrm{H}$ NMR $(400 \mathrm{MHz}$, DMSO- $\left.d_{6}\right): \delta_{\mathrm{H}} 2.27\left(3 \mathrm{H}, \mathrm{s}, \mathrm{O}-\mathrm{C}-\mathrm{CH}_{3}\right), 3.13\left(3 \mathrm{H}, \mathrm{d},{ }^{3} \mathrm{~J}_{\mathrm{HH}} 4.0 \mathrm{~Hz}, \mathrm{NH}-\mathrm{CH}_{3}\right), 5.32(1 \mathrm{H}, \mathrm{s}, \mathrm{C}-\mathrm{CH}-$ C), $6.57(1 \mathrm{H}, \mathrm{s}, \mathrm{C}=\mathrm{CH}-\mathrm{C}), 6.89-6.91(2 \mathrm{H}, \mathrm{m}, \mathrm{Ph}), 7.31-7.32(1 \mathrm{H}, \mathrm{m}, \mathrm{Ph}), 10.29\left(1 \mathrm{H}, \mathrm{d},{ }^{3} J_{\mathrm{HH}} 4.0\right.$ $\mathrm{Hz}, \mathrm{NH}) ;{ }^{13} \mathrm{C}$ NMR $\left(100 \mathrm{MHz}, \mathrm{DMSO}-d_{6}\right): \delta_{\mathrm{C}} 19.4\left(\mathrm{O}-\mathrm{C}-\mathrm{CH}_{3}\right), 28.4\left(\mathrm{NH}-\mathrm{CH}_{3}\right), 31.4(\mathrm{C}-\mathrm{CH}-\mathrm{C})$, $97.9\left(\mathrm{C}-\mathrm{NO}_{2}\right), 102.8(\mathrm{C}=\mathrm{C}-\mathrm{O}), 107.5(\mathrm{C}=\mathrm{C}-\mathrm{C}), 124.5,125.1,126.6$ and $144.8(4 \mathrm{C}$, thiophene $)$, $156.9(\mathrm{C}=\mathrm{C}-\mathrm{NH}), 157.0\left(\mathrm{CH}_{3}-\mathrm{C}-\mathrm{O}\right), 161.1(\mathrm{C}=\mathrm{O}), 163.4(\mathrm{C}=\mathrm{C}-\mathrm{O})$; HRMS (ESI) $m / z$ : calc. for: $\mathrm{C}_{14} \mathrm{H}_{12} \mathrm{~N}_{2} \mathrm{O}_{5} \mathrm{SNa}, 343.0359[\mathrm{M}+\mathrm{Na}]^{+}$; found: 343.0350 . 
4-Furan-2-yl-7-methyl-2-(methylamino)-3-nitro-4H-pyrano[4,3-b]pyran-5-one (4n). Yellow

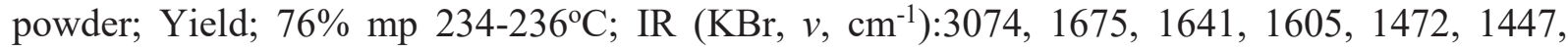
$1363,1257,1188,1139,1072,1013,890,793,771 \mathrm{~cm}^{-1}$; ${ }^{1} \mathrm{H}$ NMR (400 MHz, DMSO- $\left.d_{6}\right): \delta_{\mathrm{H}}$ $2.26\left(3 \mathrm{H}, \mathrm{s}, \mathrm{O}-\mathrm{C}-\mathrm{CH}_{3}\right), 3.12\left(3 \mathrm{H}, \mathrm{d},{ }^{3} \mathrm{~J}_{\mathrm{HH}} 4.8 \mathrm{~Hz}, \mathrm{NH}-\mathrm{CH}_{3}\right), 5.16(1 \mathrm{H}, \mathrm{s}, \mathrm{C}-\mathrm{CH}-\mathrm{C}), 6.26-6.35$ $(2 \mathrm{H}, \mathrm{m}, \mathrm{Ph}), 6.59(1 \mathrm{H}, \mathrm{s}, \mathrm{C}=\mathrm{CH}-\mathrm{C}), 7.46(1 \mathrm{H}, \mathrm{s}, \mathrm{Ph}), 10.30\left(1 \mathrm{H}, \mathrm{d},{ }^{3} J_{\mathrm{HH}} 4.8 \mathrm{~Hz}, \mathrm{NH}\right) ;{ }^{13} \mathrm{C}$ NMR

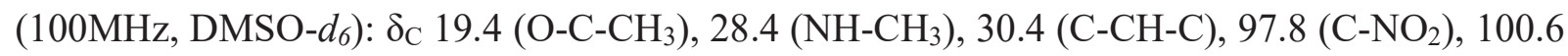
$(\mathrm{C}=\mathrm{C}-\mathrm{O}), 105.1(\mathrm{C}=\mathrm{C}-\mathrm{C}), 107.1,110.5,141.8$ and $152.1 \quad(4 \mathrm{C}$, furan), $157.1(\mathrm{C}=\mathrm{C}-\mathrm{NH}), 157.5$ $\left(\mathrm{CH}_{3}-\mathrm{C}-\mathrm{O}\right), 160.9(\mathrm{C}=\mathrm{O}), 163.4(\mathrm{C}=\mathrm{C}-\mathrm{O})$; HRMS (ESI) $m / z$ : calc. for: $\mathrm{C}_{14} \mathrm{H}_{12} \mathrm{~N}_{2} \mathrm{O}_{6} \mathrm{Na}, 327.0588$ $[\mathrm{M}+\mathrm{Na}]^{+}$; found: 327.0586 .

4-(2-Methoxyphenyl)-2-(methylamino)-3-nitro-4H-pyrano[3,2-c]chromen-5-one (6a). Light yellow powder; Yield; 69\%; mp 251-253 ${ }^{\circ} \mathrm{C}$; IR (KBr, v, $\left.\mathrm{cm}^{-1}\right): 3186,1730,1635,1470,1459$, $1381,1293,1168,1104,1053,1023,777,757,695 \mathrm{~cm}^{-1}$; ${ }^{1} \mathrm{H}$ NMR (400 MHz, DMSO- $\left.d_{6}\right): \delta_{\mathrm{H}}$ $3.35\left(3 \mathrm{H}, \mathrm{d},{ }^{3} J_{\mathrm{HH}} 5.2 \mathrm{~Hz}, \mathrm{NH}-\mathrm{CH}_{3}\right), 3.61\left(3 \mathrm{H}, \mathrm{s}, \mathrm{OCH}_{3}\right), 5.08(1 \mathrm{H}, \mathrm{s}, \mathrm{C}-\mathrm{CH}-\mathrm{C}), 6.89-6.92(2 \mathrm{H}$, $\mathrm{m}, \mathrm{Ph}), 6.93-7.23$ (1H, m, Ph), 7.39-7.55 (3H, m, Ph), 7.72-7.76 (1H, m, Ph), 8.04-8.06 (1H, m, $\mathrm{Ph}), 10.46\left(1 \mathrm{H}, \mathrm{d},{ }^{3} J_{\mathrm{HH}} 5.2 \mathrm{~Hz}, \mathrm{NH}\right) ;{ }^{13} \mathrm{C} \mathrm{NMR}\left(100 \mathrm{MHz}, \mathrm{DMSO}-d_{6}\right): \delta_{\mathrm{C}} 28.5\left(\mathrm{NH}_{-} \mathrm{CH}_{3}\right), 35.9$ (C-CH-C), $55.4\left(\mathrm{O}-\mathrm{CH}_{3}\right), 104.5\left(\mathrm{C}-\mathrm{NO}_{2}\right), 106.5(\mathrm{C}=\mathrm{C}-\mathrm{C}), 111.8,112.6,116.6,119.8,122.5$, 125.0, 126.8, 128.6, 132.9 (2C), 151.9 and $152.2(12 \mathrm{C}$, benzene), $157.6(\mathrm{C}=\mathrm{C}-\mathrm{NH}), 157.7(\mathrm{C}=\mathrm{C}-$ O), $159.2(\mathrm{C}=\mathrm{O})$; HRMS (ESI) $m / z$ : calc. for: $\mathrm{C}_{20} \mathrm{H}_{16} \mathrm{~N}_{2} \mathrm{O}_{6} \mathrm{Na}, 403.0926[\mathrm{M}+\mathrm{Na}]^{+}$; found:403.0922.

4-(4-Dimethylaminophenyl)-2-(methylamino)-3-nitro-4H-pyrano[3,2-c]chromen-5-one (6b). Orange powder; Yield; 74\%; mp 259-261 ${ }^{\circ} \mathrm{C}$; IR (KBr, v, $\left.\mathrm{cm}^{-1}\right): 3209,3074,1736,1675$, 1609,1522, 1456, 1356, 1159, 1106, 1071, 785, 771, $687 \mathrm{~cm}^{-1}$; ${ }^{1} \mathrm{H}$ NMR (400 MHz, DMSO- $\left.d_{6}\right)$ : $\delta_{\mathrm{H}} 2.82\left(6 \mathrm{H}, \mathrm{s}, 2 \mathrm{CH}_{3}\right), 3.32\left(3 \mathrm{H}, \mathrm{d},{ }^{3} J_{\mathrm{HH}} 4.8 \mathrm{~Hz}, \mathrm{NH}-\mathrm{CH}_{3}\right), 4.97(1 \mathrm{H}, \mathrm{s}, \mathrm{C}-\mathrm{CH}-\mathrm{C}), 6.60(2 \mathrm{H}, \mathrm{d}$, $\left.{ }^{3} J_{\mathrm{HH}} 8.8 \mathrm{~Hz} 2 \mathrm{H}, \mathrm{Ph}\right), 7.11\left(2 \mathrm{H}, \mathrm{d},{ }^{3} J_{\mathrm{HH}} 8.8 \mathrm{~Hz}, \mathrm{Ph}\right), 7.48-7.53(2 \mathrm{H}, \mathrm{m}, \mathrm{Ph}), 7.71-7.75(1 \mathrm{H}, \mathrm{m}$, $\mathrm{Ph}), 7.99-8.01(1 \mathrm{H}, \mathrm{m}, \mathrm{Ph}), 10.36\left(1 \mathrm{H}, \mathrm{d},{ }^{3} J_{\mathrm{HH}} 5.2 \mathrm{~Hz}, \mathrm{NH}\right) ;{ }^{13} \mathrm{C} \mathrm{NMR}\left(100 \mathrm{MHz}, \mathrm{DMSO}-d_{6}\right): \delta_{\mathrm{C}}$ $28.7\left(\mathrm{NH}-\mathrm{CH}_{3}\right), 36.9(\mathrm{C}-\mathrm{CH}-\mathrm{C}), 43.5\left(2 \mathrm{C}, 2 \mathrm{CH}_{3}\right), 106.3\left(\mathrm{C}-\mathrm{NO}_{2}\right), 107.7(\mathrm{C}=\mathrm{C}-\mathrm{C}), 112.6,116.6$ (2C), $122.8(2 \mathrm{C}), 125.0(2 \mathrm{C}), 129.7(2 \mathrm{C}), 133.2(2 \mathrm{C})$ and 151.8 (12C, benzene), $152.0(\mathrm{C}=\mathrm{C}-$ $\mathrm{NH}), 156.7(\mathrm{C}=\mathrm{C}-\mathrm{O}), 159.2(\mathrm{C}=\mathrm{O})$; HRMS (ESI) $m / z$ : calc. for: $\mathrm{C}_{21} \mathrm{H}_{19} \mathrm{~N}_{3} \mathrm{O}_{5} \mathrm{Na}, 416.1325$ $[\mathrm{M}+\mathrm{Na}]^{+}$; found:416.1219.

2-(Methylamino)-3-nitro-4-thiophen-2-yl-4H-pyrano[3,2-c]chromen-5-one (6c). White powder; Yield; $75 \%$; mp 256-258 ${ }^{\circ} \mathrm{C}$; IR $\left(\mathrm{KBr}, v, \mathrm{~cm}^{-1}\right)$ : 3050, 1728, 1671, 1627, 1525, 1471, $1356,1305,1268,1163,1073,779,759,690 \mathrm{~cm}^{-1}$; ${ }^{1} \mathrm{H}$ NMR (400 MHz, DMSO-d $\left.d_{6}\right): \delta_{\mathrm{H}} 3.31(3 \mathrm{H}$, d, $\left.{ }^{3} J_{\mathrm{HH}} 5.2 \mathrm{~Hz}, \mathrm{NH}-\mathrm{CH}_{3}\right), 5.48(1 \mathrm{H}, \mathrm{s}, \mathrm{C}-\mathrm{CH}-\mathrm{C}), 6.90-6.99(2 \mathrm{H}, \mathrm{m}, \mathrm{Ph}), 7.34-7.36(1 \mathrm{H}, \mathrm{m}, \mathrm{Ph})$, 7.50-7.55 (2H, m, Ph), 7.75-7.79 (1H, m, Ph), 8.00-8.02 (1H, m, Ph), $10.38\left(1 \mathrm{H}, \mathrm{d},{ }^{3} J_{\mathrm{HH}} 5.2 \mathrm{~Hz}\right.$, $\mathrm{NH}) ;{ }^{13} \mathrm{C}$ NMR (100MHz, DMSO- $\left.d_{6}\right): \delta_{\mathrm{C}} 28.7\left(\mathrm{NH}-\mathrm{CH}_{3}\right), 32.0(\mathrm{C}-\mathrm{CH}-\mathrm{C}), 106.2\left(\mathrm{C}-\mathrm{NO}_{2}\right), 107.5$ $(\mathrm{C}=\mathrm{C}-\mathrm{C}), 112.5,116.7,122.8,124.8,125.1,125.4,126.7,133.4,144.2$ and $152.0(10 \mathrm{C}$, benzene and thiophene), $152.3(\mathrm{C}=\mathrm{C}-\mathrm{NH}), 156.7(\mathrm{C}=\mathrm{C}-\mathrm{O}), 159.2(\mathrm{C}=\mathrm{O})$; HRMS (ESI) $m / z$ : calc. for: $\mathrm{C}_{17} \mathrm{H}_{12} \mathrm{~N}_{2} \mathrm{O}_{5} \mathrm{SNa}, 379.0365[\mathrm{M}+\mathrm{Na}]^{+}$; found:379.0357.

4-Furan-2-yl-2-(methylamino)-3-nitro-4H-pyrano[3,2-c]chromen-5-one (6d). White powder; Yield; 82\%; mp 258-260 $\mathrm{C}$; IR (KBr, $\left.v, \mathrm{~cm}^{-1}\right): 3137,1725,1632,1472,1455,1380,1304,1267$, 
$1156,1106,1077,779,768,706 \mathrm{~cm}^{-1} ;{ }^{1} \mathrm{H}$ NMR (400 MHz, DMSO- $\left.d_{6}\right): \delta_{\mathrm{H}} 3.30\left(3 \mathrm{H}, \mathrm{d},{ }^{3} J_{\mathrm{HH}} 4.0\right.$ $\left.\mathrm{Hz}, \mathrm{NH}-\mathrm{CH}_{3}\right), 5.33$ (1H, s, C-CH-C), 6.33-6.37 (2H, m, Ph), 7.47-7.53 (3H, m, Ph), 7.74-7.78 $(1 \mathrm{H}, \mathrm{m}, \mathrm{Ph}), 7.99-8.00(1 \mathrm{H}, \mathrm{m}, \mathrm{Ph}), 10.37\left(1 \mathrm{H}, \mathrm{d},{ }^{3} J_{\mathrm{HH}} 4.4 \mathrm{~Hz}, \mathrm{NH}\right) ;{ }^{13} \mathrm{C} \mathrm{NMR}(100 \mathrm{MHz}$, DMSO- $\left.d_{6}\right): \delta_{\mathrm{C}} 28.7\left(\mathrm{NH}-\mathrm{CH}_{3}\right), 31.1(\mathrm{C}-\mathrm{CH}-\mathrm{C}), 104.1\left(\mathrm{C}-\mathrm{NO}_{2}\right), 105.1(\mathrm{C}=\mathrm{C}-\mathrm{C}), 107.4,110.6$, $112.5,116.7,122.8,125.1,133.4,142.1,151.8$ and 152.0 (10C, benzene and furan), $152.8(\mathrm{C}=\mathrm{C}-$ $\mathrm{NH}), 157.0(\mathrm{C}=\mathrm{C}-\mathrm{O}), 159.1(\mathrm{C}=\mathrm{O})$; HRMS (ESI) $m / z$ : calc. for: $\mathrm{C}_{17} \mathrm{H}_{12} \mathrm{~N}_{2} \mathrm{O}_{5} \mathrm{ONa}, 363.0607$ $[\mathrm{M}+\mathrm{Na}]^{+}$; found:363.0605.

\section{Supplementary Material}

The materials of the structural characterization with of ${ }^{1} \mathrm{H}$ NMR, ${ }^{13} \mathrm{C}$ NMR and IR for this article are available online at the supplementary materials.

\section{Acknowledgements}

The authors thank the National Natural Science Funds of China (No. 21106069).

\section{References}

1. Green, G. R.; Evans, J. M.; Vong, A. K. In Comprehensive Heterocyclic Chemistry II; Katritzky, A. R.; Rees, C. W.; Scriven, E. F. V. Eds.; Pergamon Press: Oxford, 1995; Vol. 5, p 469.

2. Konecny, U. M.; Rajniakova, V. O. Chem. Pap. 1994, 48, 282.

3. Balzarini, P. P. M J.; Rozenski, J.; Clercq, D.; Herdewijn, P. Bioorg. Med. Chem. Lett. 1995, 5,1115 .

4. Takao, H.; Murai, K.; Yasudomi, N.; Goto, T.; Umetsu, N.; Horie, T. J. Pestic. Sci. 1994, 19, 131.

http://dx.doi.org/10.1584/jpestics.19.3_151

5. Leutbecher, H.; Williams, L. A. D.; Rosner, H.; Beifuss, U. Bioorg. Med. Chem. Lett. 2007, 17,978 .

http://dx.doi.org/10.1016/j.bmcl.2006.11.045

PMid:17166717

6. Chung, H. S.; Kim, Y.; Oh, S. J.; Kim, H.; Choi, S. I.; Zhang, Y. J.; Jeong, J. H.; Bae, H.

Bioorg. Med. Chem. 2013, 21, 6359.

http://dx.doi.org/10.1016/j.bmc.2013.04.004

http://dx.doi.org/10.1016/j.bmc.2013.08.045

PMid:24054491 
7. Fan, X. S.; Feng, D.; Qu, Y. Y.; Zhang, X. Y.; Wang, J. J.; Loiseau, P. M.; Andrei, G.; Snoeck, R.; Clercq, E. D. Bioorg. Med. Chem. Lett. 2010, 20, 809. http://dx.doi.org/10.1584/jpestics.19.3_151

8. Parthasarathy, K.; Praveen, C.; Balachandran, C.; Senthilkumar, P.; Ignacimuthu, S.; Perumal, P. T. Bioorg. Med. Chem. Lett. 2013, 20, 2708.

http://dx.doi.org/10.1016/j.bmcl.2013.02.086

PMid:23522833

9. Christian, P. R.; Chaitan K. ACS Chem. Biol. 2007, 2, 104. http://dx.doi.org/10.1021/cb600382j

PMid:17256996

10. Xia, X. F.; Shu, X. Z.; Ji, K. G.; Shaukat, A.; Liu, X. Y.; Liang, Y. M. J. Org. Chem. 2011, 76,342 .

http://dx.doi.org/10.1021/jo102219z http://dx.doi.org/10.1021/jo201253m

11. Volker, B. F.; Helmut, G.; Rolf-Eberhard, N. J. Cardiovasc. Pharm. 1985, 7, 666. http://dx.doi.org/10.1097/00005344-198507000-00009

12. Pastine, S. J.; Youn, S. W.; Sames, D. Org. Lett. 2003, 5, 1055. http://dx.doi.org/10.1021/o1034177k http://dx.doi.org/10.1021/o1035419j

13. Khalilzadeh, M. A.; Hossaini, Z. S.; Baradarani, M.; Hasanni, A. Tetrahedron 2010, 66, 8464. http://dx.doi.org/10.1016/j.tet.2010.08.041

14. Devi, I.; Bhuyan, P. J. Tetrahedron Lett. 2004, 45, 8625. http://dx.doi.org/10.1016/j.tetlet.2004.09.158 http://dx.doi.org/10.1016/j.tetlet.2004.01.094 http://dx.doi.org/10.1016/j.tetlet.2004.08.072

15. Heravi, L. F.; Fatehi, M. M.; Bakhtiari, A. K. Tetrahedron Lett. 2007, 48, 5379. http://dx.doi.org/10.1016/j.tetlet.2007.06.035

16. Jin, T. S.; Wang, A.Q.; Wang, X.; J. Zhang, S.; Li, T. S. Synlett. 2005, 35, 137.

17. Moghadam, K. R.; Kiasaraie, M. S.; Azimi, S.C. Tetrahedron 2012, 68, 6472. http://dx.doi.org/10.1016/j.tet.2012.05.113

18. Kamalraja, J.; Muralidharan, D.; Perumal, P. T. Synlett. 2012, 23, 2894. http://dx.doi.org/10.1055/s-0032-1317543

19. Ziarani, G. M.; Badiei, S. A.; Mousavi, S.; Gholamzadeh, P. Res. Chem. Intermed. 2015, 41, 637. http://dx.doi.org/10.1007/s11164-013-1217-x

20. Mosaddegh, E.; Hassankhani, A. Chin. J. Cat. 2014, 35, 351. http://dx.doi.org/10.1016/S1872-2067(12)60755-4 
21. Nguyen, N. T.; Greenhalgh, E.; Kamaruddin, M. J.; Elharfi, J.; Carmichael, K.; Dimitrakis, G.; Kingman, S. W.; Robinson, J. P.; Irvine. D. J. Tetrahedron 2014, 70, 996. http://dx.doi.org/10.1016/j.tet.2013.11.031

22. Tu, S. J.; Jiang, B.; Zhang, J. Y.; Jia, R. H.; Zhang, Y; Yao, C. S. Org. Biomol. Chem. 2006, 4, 3980 .

http://dx.doi.org/10.1039/b604218j http://dx.doi.org/10.1039/b607575d http://dx.doi.org/10.1039/b611462h PMid:17047879

23. Lv, L. Y.; Zheng, S. C.; Cai, X. T.; Chen, Z. P.; Zhu, Q. H.; Liu, S. W. ACS Comb. Sci. 2013, 15,183 .

24. http://dx.doi.org/10.1021/co300148c

PMid:23425098

25. Che, C.; Li, S.; Yu, Z.; Li, F.; Xin, S.; Zhou, L.; Lin, S.; Yang, Z. ACS Comb. Sci. 2013, 15, 202.

26. http://dx.doi.org/10.1021/co400001h PMid:23441930

27. Zhang, J.J.; Feng, X.; Liu, X. C.; Huang, Z. B.; Shi, D. Q. Mol. Divers 2014, 18, 727. http://dx.doi.org/10.1007/s11030-014-9544-4 http://dx.doi.org/10.1007/s11030-013-9487-1 http://dx.doi.org/10.1007/s11030-014-9538-2 http://dx.doi.org/10.1007/s11030-013-9495-1

28. Suresh, L.; Poornachandra, Y.; Kanakaraju, S.; Kumar, C. G.; Chandramouli, G. V. P. Org. Biomol. Chem. 2015, 13, 7294. http://dx.doi.org/10.1039/C5OB00693G PMid:26054925

29. Kurosh, R. M.; Masoumeh, S. K.; Seyyedeh, C. A. Tetrahedron 2012, 68, 6472. http://dx.doi.org/10.1016/j.tet.2012.05.113

30. Ipsita, D.; Pulak, J. B. Tetrahedron Lett. 2004, 45, 8625. http://dx.doi.org/10.1016/j.tetlet.2004.09.158

31. Kaur, P.; Pindi, S.; Wever, W.; Rajale, T.; Li, G. Chem. Commun. 2010, 46, 4330. http://dx.doi.org/10.1039/c0cc00287a PMid:20464009

32. Hui-Yan, W.; Da-Qing, S. ACS. Comb. Sci. 2013, 15, 261. http://dx.doi.org/10.1021/co4000198 PMid:23597107

33. Kaur, P.; Wever, W.;Pindi, S.; Milles, R.; Gu, P.; Shi, M.; Li, G. Green Chem. 2011,13, 1288. http://dx.doi.org/10.1039/c1gc15029d 